\title{
Performance Analysis for Multichannel Reception of OOFSK Signaling
}

\author{
Qingyun Wang Mustafa Cenk Gursoy \\ Department of Electrical Engineering \\ University of Nebraska-Lincoln, Lincoln, NE 68588 \\ Email: qwang4@bigred.unl.edu, gursoy@unl.edu
}

\begin{abstract}
In this paper, the error performance of onoff frequency shift keying (OOFSK) modulation over fading channels is analyzed when the receiver is equipped with multiple antennas. The analysis is conducted in two cases: the coherent scenario where the fading is perfectly known at the receiver, and the noncoherent scenario where neither the receiver nor the transmitter knows the fading coefficients. For both cases, the maximum a posteriori probability (MAP) detection rule is derived and analytical probability of error expressions are obtained. The effect of fading correlation among the receiver antennas is also studied. Simulation results indicate that for sufficiently low duty cycle values, lower probability of error values with respect to FSK signaling are achieved. Equivalently, when compared to FSK modulation, OOFSK with low duty cycle requires less energy to achieve the same probability of error, which renders this modulation a more energy efficient transmission technique.
\end{abstract}

\section{INTRODUCTION}

Frequency-shift keying (FSK) is a modulation format that is well-known and well-studied in the communications literature [15]. FSK is an attractive transmission scheme due to its high energy efficiency and suitability for noncoherent communications. In unknown channel conditions, energy detection can be employed to detect the FSK signals. Indeed, the analysis of FSK modulation dates back to 1960s (see e.g., [1], and [2]). Recently, it has been shown in [5] that unless the channel conditions are perfectly known at the receiver, signals that have very high peak-to-average power ratio is required to achieve the capacity in the low SNR regime. This has initiated work on peaky signaling. Luo and Médard [6] have shown that FSK with small duty cycle can achieve rates of the order of capacity in ultrawideband systems with limits on bandwidth and peak power. In [8], the authors have studied the error performance of peaky FSK signaling over multipath fading channels by obtaining upper and lower bounds on the error probability. In [9], on-off frequency-shift keying (OOFSK) is defined as FSK overlaid on on-off keying, and its capacity and energy efficiency is analyzed. Note that OOFSK can be seen as joint pulse position modulation (PPM) and FSK. In this signaling, peakedness is introduced in both time and frequency. The error performance of OOFSK signaling when the transmitter and receiver are each equipped with a single antenna is recently studied in [10].

\footnotetext{
${ }^{1}$ This work was supported in part by the NSF CAREER Grant CCF0546384.
}

One of the important techniques to improve the performance in wireless communications is to use multiple antennas to achieve diversity gain. Considerable amount of work has been done on multiple reception channels. In [2], it is shown for binary and $M$-ary signaling over Rician fading channels that increasing the number of reception channels can improve the error performance significantly. By finding the probability distribution function of the instantaneous SNR in flat fading multi-reception channels and substituting it into the probability of error expressions of PAM, PSK and QAM over AWGN channel, the authors in [12] obtained expressions for the average probability of error of multi-reception fading channels. In [14], the probability of error of BPSK over Rician fading multi-reception channels is given and extensions to other modulation techniques are discussed. In [17], average symbol error rate of selection diversity of $M$-ary FSK modulated signal transmitted over fading channels is studied.

In this paper, the error performance of OOFSK over multiple reception Rician fading channels is studied. In Section [II, the system model is presented. In Section III the error performance in coherent fading channels is studied. In Section [V] we investigate the error performance in noncoherent Rician fading channels.

\section{SySTEM MODEL}

We assume that OOFSK modulation is employed at the transmitter to send the information. In OOFSK modulation, the transmitted signal during the symbol interval $0 \leq t \leq T_{s}$ can be expressed as

$$
s_{m}(t)= \begin{cases}\sqrt{\frac{P}{v}} e^{j\left(w_{m} t+\theta_{m}\right)} & m=1,2,3, \ldots M \\ 0 & m=0\end{cases}
$$

where $w_{m}$ and $\theta_{m}$ are the frequency in radians per second and phase, respectively, of the signal $s_{m}(t)$ when $m \neq 0$. Note that we have $M$ FSK signals and a zero signal denoted by $s_{0}(t)$. The frequencies of the FSK signals are chosen so that the signals are orthogonal. It is assumed that an FSK signal $s_{m}(t), m \neq 0$, is transmitted with a probability of $\frac{v}{M}$ while $s_{0}(t)$ is transmitted with a probability of $1-v$ where $v$ is the duty cycle of transmission. With these definitions, it is easily seen that $P$ and $\frac{P}{v}$ are the average and peak powers, respectively, of the modulation technique.

The receiver is equipped with $L$ antennas that enable the multiple reception of the transmitted signal. If, without loss 
of generality, we assume that $s_{k}(t)$ is the transmitted signal, the received signal at the $l^{\text {th }}$ antenna is

$$
r_{l}(t)=h_{l} s_{k}(t)+n_{l}(t) \quad l=1,2, \ldots, L
$$

where $h_{l}$ is the fading coefficient at the $l^{\text {th }}$ reception channel and $n_{l}(t)$ is a white Gaussian noise with single-sided spectral density of $N_{0}$. It is assumed that the additive Gaussian noise components at different antennas are independent. Furthermore, the received signal model (2) presumes that the fading is frequency-flat and slow enough so that the fading coefficient stays constant over one symbol duration.

Following each antenna, there is a bank of $M$ correlators, each correlating the received signal with one of the orthogonal frequencies. The output of the $m^{\text {th }}$ correlator employed after the $l^{\text {th }}$ antenna is given by

$$
\begin{aligned}
Y_{l, m} & =\frac{1}{\sqrt{N_{0} T_{s}}} \int_{0}^{T_{s}} r_{l}(t) e^{-j w_{m} t} d t \\
& = \begin{cases}\sqrt{\frac{P T_{s}}{v N_{0}}} h_{l} e^{j \theta_{m}}+n_{l, m} & m=k \\
n_{l, m} & m \neq k\end{cases} \\
& = \begin{cases}A h_{l} e^{j \theta_{m}}+n_{l, m} & m=k \\
n_{l, m} & m \neq k,\end{cases}
\end{aligned}
$$

where $n_{l, m}$ is a circularly symmetric complex Gaussian random variable with zero-mean and a variance of 1 and for notational convenience, we have defined $A=\sqrt{\frac{P T_{s}}{v N_{0}}}$. Since the frequencies are orthogonal and the additive Gaussian noise is independent at each antenna, $\left\{n_{l, m}\right\}$ for $l \in\{1, \ldots, L\}$ and $m \in\{1, \ldots, M\}$ forms an independent and identically distributed (i.i.d.) sequence. Note also that $R_{l, m}=\left|Y_{l, m}\right|^{2}$ gives the energy present in the $m^{\text {th }}$ frequency at the $l^{\text {th }}$ antenna.

\section{OOFSK Over COHERENT FADING ChanNELS}

\section{A. Detection Rule}

In this section, we assume that transmission takes place over coherent fading channels and hence $h_{l}$ for all $l$ is known to the receiver while the transmitter does not have such knowledge. Conditioned on $h_{l}$ and the transmitted signal $s_{k}(t), Y_{l, m}$ is a proper complex Gaussian random variable with mean value and variance given by

$$
\begin{aligned}
& E\left\{Y_{l, m} \mid h_{l}, s_{k}\right\}= \begin{cases}A h_{l} e^{j \theta_{k}} & m=k \\
0 & m \neq k\end{cases} \\
& \operatorname{var}\left\{Y_{l, m} \mid h_{l}, s_{k}\right\}=1 .
\end{aligned}
$$

Therefore, $R_{l, m}=\left|Y_{l, m}\right|^{2}$ is chi-square distributed with the following conditional probability density function (pdf):

$f_{R_{l, m}|| h_{l} \mid, s_{k}}\left(R_{l, m}\right)= \begin{cases}e^{-\left(R_{l, m}+A^{2}\left|h_{l}\right|^{2}\right)} I_{0}\left(2 A\left|h_{l}\right| \sqrt{R_{l, m}}\right) & m=k \\ e^{-R_{l, m}} & m \neq k\end{cases}$

It is assumed that the receiver, using equal gain combining (EGC), combines the energies of the $m^{\text {th }}$ frequency components at each antenna, i.e., computes the total energy

$$
R_{m}=\sum_{l=1}^{L} R_{l, m}
$$

Since the noise components are independent, $R_{m}$ is a sum of independent chi-square random variables, and is itself also chisquare distributed with $2 L$ degrees of freedom. The conditional pdf is given by

$f_{R_{m} \mid \mathbf{h}, s_{k}}\left(R_{m}\right)= \begin{cases}\left(\frac{R_{m}}{\xi}\right)^{\frac{L-1}{2}} e^{-\left(R_{m}+\xi\right)} I_{L-1}\left(2 \sqrt{R_{m} \xi}\right) & m=k \\ \frac{R_{m}^{L-1}}{\Gamma(L)} e^{-R_{m}} & m \neq k\end{cases}$

where $\xi=\sum_{l=1}^{L} A^{2}\left|h_{l}\right|^{2}, \mathbf{h}=\left[h_{1}, \ldots, h_{L}\right], I_{L-1}(\cdot)$ is the $(L-1)^{\text {th }}$ order modified Bessel function of the first kind, and $\Gamma(\cdot)$ is the gamma function.

The receiver employs maximum a posteriori probability (MAP) criterion to detect the transmitted signals. Let

$$
\mathbf{R}=\left[R_{1}, R_{2}, \ldots, R_{M}\right]
$$

be the vector of energy values corresponding to each frequency. Since the noise components $n_{l, m}$ are independent for different $m \in\{1, \ldots, M\}$, components of $\mathbf{R}$ are mutually independent. Hence, the conditional pdf of $\mathbf{R}$ is

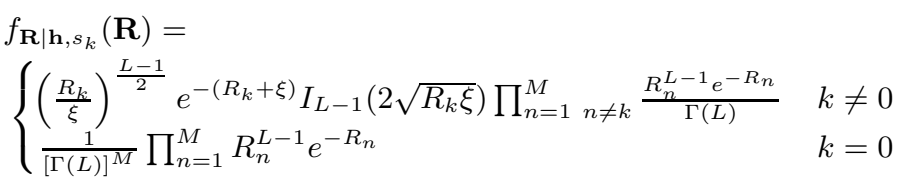

Then, the MAP rule that detects $s_{k}$ for $k \neq 0$ is

$$
\left\{\begin{array}{l}
f_{\mathbf{R} \mid \mathbf{h}, s_{k}}>f_{\mathbf{R} \mid \mathbf{h}, s_{m}} \\
f_{\mathbf{R} \mid \mathbf{h}, s_{k}}>\frac{M(1-v)}{v} f_{\mathbf{R} \mid \mathbf{h}, s_{0}}
\end{array} \quad \forall m \neq 0, k\right.
$$

where we have used the fact that the prior probabilities of the transmitted signals are $p\left(s_{m}\right)=\frac{v}{M}$ for $m \neq 0$, and $p\left(s_{0}\right)=(1-v)$. Substituting (7) into to (8), the decision rule is simplified to:

$$
\left\{\begin{array}{l}
g_{1}\left(R_{k}\right)>g_{1}\left(R_{m}\right) \quad \forall m \neq k \\
g_{1}\left(R_{k}\right)>\frac{M(1-v) e^{\xi} \xi^{\frac{L-1}{2}}}{v(L-1) !}
\end{array} \quad \forall\right.
$$

where

$$
g_{1}\left(R_{k}\right)=R_{k}^{-\frac{L-1}{2}} I_{L-1}\left(2 \sqrt{R_{k} \xi}\right), \quad \xi>0 .
$$

The following Lemma enables us to further simplify the detection rule.

Lemma 1: The function

$$
g_{1}(x)=x^{-\frac{L-1}{2}} I_{L-1}(2 \sqrt{x \xi}) \text { for } x>0, \xi>0
$$

is a monotonically increasing function of $x$.

Proof: The derivative of the $n^{\text {th }}$ order modified Bessel function is

$$
\frac{d I_{n}(x)}{d x}=I_{n+1}(x)+\frac{n}{x} I_{n}(x) .
$$

Hence,

$$
\begin{aligned}
\frac{d I_{L-1}(2 \sqrt{x \xi})}{d x} & =\sqrt{\frac{\xi}{x}} I_{L}(2 \sqrt{x \xi})+\frac{L-1}{2 x} I_{L-1}(2 \sqrt{x \xi}) \\
& >\frac{L-1}{2 x} I_{L-1}(2 \sqrt{x \xi})
\end{aligned}
$$

where we use the fact that $\sqrt{\frac{\xi}{x}} I_{L}(2 \sqrt{x \xi})>0$ for $x>0$. 
Then, the derivative of $g_{1}(\cdot)$ satisfies

$$
\begin{aligned}
& \frac{d g_{1}(x)}{d x}=-\frac{L-1}{2} x^{-\frac{L+1}{2}} I_{L-1}(2 \sqrt{x \xi})+x^{-\frac{L-1}{2}} \frac{d I_{L-1}(2 \sqrt{x \xi})}{d x} \\
& >-\frac{L-1}{2} x^{-\frac{L+1}{2}} I_{L-1}(2 \sqrt{x \xi})+x^{-\frac{L-1}{2}} \frac{L-1}{2 x} I_{L-1}(2 \sqrt{x \xi}) \\
& =0,
\end{aligned}
$$

proving that $g_{1}(x)$ is a monotonically increasing function of $x>0$.

By the above result, the detection rule (9) further simplifies to

$$
\left\{\begin{array}{l}
R_{k}>R_{m} \\
R_{k}>g_{1}^{-1}(T)
\end{array} \quad \forall m \neq k\right.
$$

where $T=\frac{M(1-v) e^{\xi} \xi^{\frac{L-1}{2}}}{v(L-1) !}$. Since $g_{1}(\cdot)$ is a monotonically increasing function, the inverse $g_{1}^{-1}(\cdot)$ is well-defined. Note that (14) is the rule that detects the signal $s_{k}(t)$ for $k \neq 0$. The zero signal $s_{0}(t)$ is detected if

$$
R_{k}<g_{1}^{-1}(T) \quad \forall k
$$

\section{B. Probability of Error}

In this section, we analyze the error probability of OOFSK modulation when MAP detection is used at the receiver. Suppose without loss of generality that $s_{1}(t)$ is the transmitted signal. Let $\tau=g_{1}^{-1}(T)$. Then the correct detection probability is

$$
\begin{aligned}
P_{c, 1} & =P\left(R_{1}>R_{2}, R_{1}>R_{3}, \ldots, R_{1}>R_{M}, R_{1}>\tau \mid s_{1}\right) \\
& =\int_{\tau}^{\infty}\left(\int_{0}^{x} f_{R_{2} \mid \mathbf{h}, s_{1}}(t) d t\right)^{M-1} f_{R_{1} \mid \mathbf{h}, s_{1}}(x) d x \\
& =\int_{\tau}^{\infty}\left(\int_{0}^{x} \frac{t^{L-1}}{\Gamma(L)} e^{-t} d t\right)^{M-1} f_{R_{1} \mid \mathbf{h}, s_{1}}(x) d x .
\end{aligned}
$$

From [15], we have

$$
\int_{0}^{x} \frac{1}{\Gamma(L)} t^{L-1} e^{-t} d t=1-e^{-x} \sum_{l=0}^{L-1} \frac{x^{l}}{l !}
$$

Therefore, the correct detection probability can now be expressed as

$$
P_{c, 1}=\int_{\tau}^{\infty}\left[1-e^{-x} \sum_{l=0}^{L-1} \frac{x^{l}}{l !}\right]^{M-1} f_{R_{1} \mid \mathbf{h}, s_{1}}(x) d x
$$

Using the binomial theorem, $P_{c, 1}$ becomes

$$
P_{c, 1}=\int_{\tau}^{\infty} \sum_{n=0}^{M-1}(-1)^{n}\left(\begin{array}{c}
M-1 \\
n
\end{array}\right)\left[\sum_{l=0}^{L-1} \frac{x^{l}}{l !} e^{-x}\right]^{n} f_{R_{1} \mid \mathbf{h}, s_{1}}(x) d x
$$

Using the multinomial theorem, we have the following expansion

$$
\left[\sum_{l=0}^{L-1} \frac{x^{l}}{l !} e^{-x}\right]^{n}=e^{-n x} \sum_{i=0}^{n(L-1)} c_{i n} x^{i}
$$

where $c_{i n}$ is the coefficient of $x^{i}$ in the expansion. $c_{i n}$ can be evaluated from the recursive equation [18]

$$
c_{i n}=\sum_{q=i-L+1}^{i} \frac{c_{q(n-1)}}{(i-q) !} I_{[0,(n-1)(L-1)]}(q)
$$

where

$$
I_{[a, b]}(q)=\left\{\begin{array}{ll}
1, & a \leq q \leq b \\
0, & \text { otherwise }
\end{array} .\right.
$$

Using the multinomial expansion, $P_{c, 1}$ becomes

$$
\begin{aligned}
P_{c, 1}= & \sum_{n=0}^{M-1}(-1)^{n}\left(\begin{array}{c}
M-1 \\
n
\end{array}\right) \sum_{i=0}^{n(L-1)} c_{i n} \int_{\tau}^{\infty} x^{i}\left(\frac{x}{\xi}\right)^{\frac{L-1}{2}} \\
& \times e^{-[(n+1) x+\xi]} I_{L-1}(2 \sqrt{x \xi}) d x
\end{aligned}
$$

Let $\xi=a^{2}$ and $x=t^{2}$, then, $P_{c, 1}$ can be written as

$$
\begin{aligned}
& P_{c, 1}=\sum_{n=0}^{M-1}(-1)^{n}\left(\begin{array}{c}
M-1 \\
n
\end{array}\right) \sum_{i=0}^{n(L-1)} c_{i n} \\
& \times \int_{\sqrt{\tau}}^{\infty} t^{2 i} e^{-n t^{2}}\left(\frac{t}{a}\right)^{L-1} e^{-\left(t^{2}+a^{2}\right)} I_{L-1}(2 a t) 2 t d t \\
& =\sum_{n=0}^{M-1}(-1)^{n}\left(\begin{array}{c}
M-1 \\
n
\end{array}\right) \sum_{i=0}^{n(L-1)} 2 c_{i n} e^{a^{2}} a^{-(L-1)} \\
& \times\left[\frac{a^{L-1} \Gamma(i+L)}{2(n+1)^{i+L} \Gamma(L)} e^{\frac{a^{2}}{n+1}} F\left(-i, L ; \frac{-a^{2}}{n+1}\right)\right. \\
& \left.-\int_{0}^{\sqrt{\tau}} t^{2 i+L} e^{-(n+1) t^{2}} I_{L-1}(2 a t) d t\right]
\end{aligned}
$$

where $F(a, c ; x)$ is the confluent hypergeometric function [16, Chap 10]. The probability of correct detection when signal $s_{0}(t)$ is transmitted is:

$$
\begin{aligned}
P_{c, 0} & =P\left(R_{1}<\tau, \ldots, R_{M}<\tau \mid \mathbf{h}, s_{0}\right) \\
& =\left(1-e^{-\tau} \sum_{l=0}^{L-1} \frac{\tau^{l}}{l !}\right)^{M} .
\end{aligned}
$$

Hence, the probability of error as a function of the instantaneous signal-to-noise ratio is

$$
P_{e}=1-\left(v P_{c, 1}+(1-v) P_{c, 0}\right) .
$$

Since the channel is assumed to be known, error probability in (23) is a function of the fading coefficients through $\chi=$ $\sum_{l=1}^{L}\left|h_{l}\right|^{2}$. Hence, the average probability of error is obtained by computing

$$
\bar{P}_{e}=\int_{0}^{\infty} P_{e} f_{\chi}(\chi) d \chi
$$

If $h_{l}$ is a complex Gaussian random variable with mean value $d_{l}$ and variance $\sigma^{2}$ and $\left\{h_{l}\right\}$ are mutually independent, $\chi$ is a chi-square random variable with $2 L$ degrees of freedom and has a pdf given by

$$
f_{\chi}(\chi)=\frac{1}{\sigma^{2}}\left(\frac{\chi}{s^{2}}\right)^{\frac{L-1}{2}} e^{-\frac{\chi+s^{2}}{\sigma^{2}}} I_{L-1}\left(\frac{2 \sqrt{\chi s^{2}}}{\sigma^{2}}\right)
$$




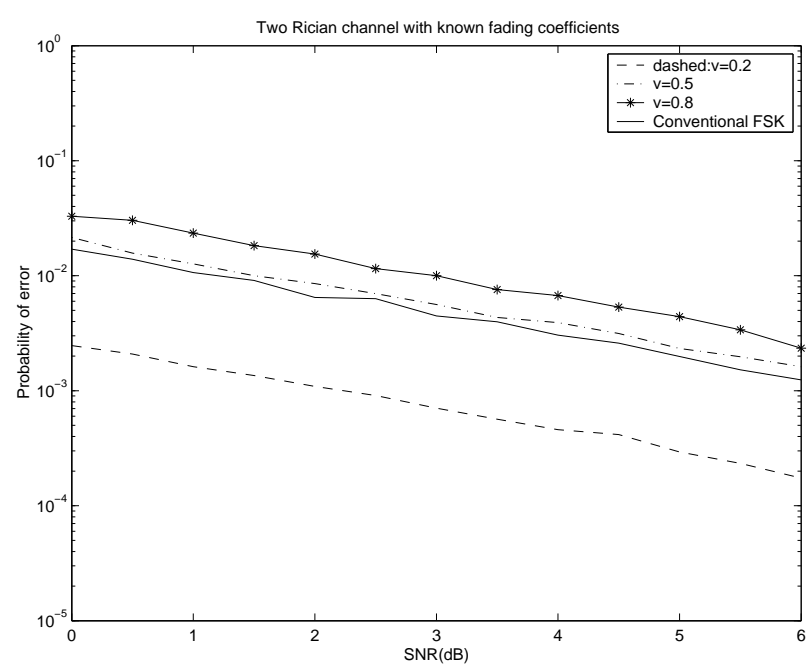

Fig. 1. Error probability vs. SNR for 4-OOFSK signaling over two independent coherent Rician fading channels with equal Rician factor $K=\frac{1}{8}$. Duty factor values are $v=1,0.8,0.5$, and 0.2 .

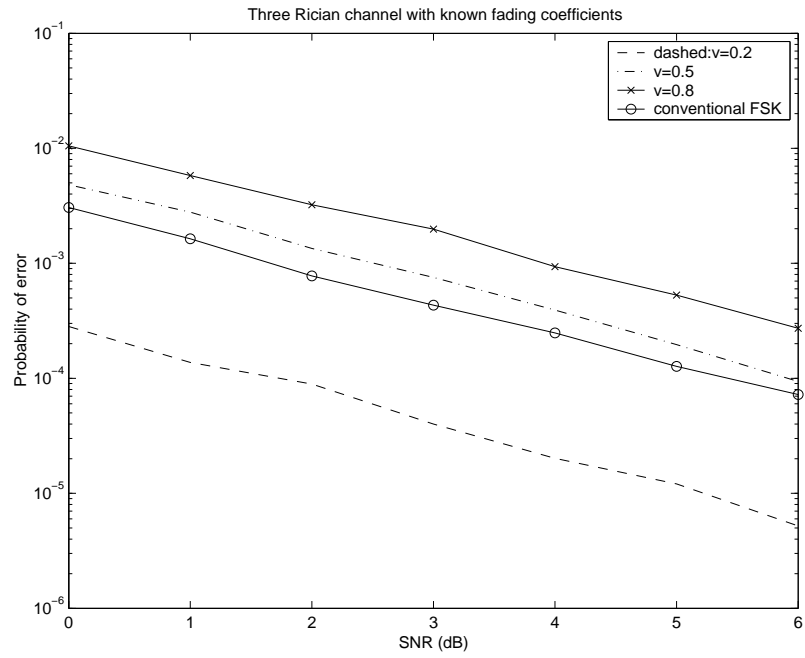

Fig. 2. Error probability vs. SNR for 4-OOFSK signaling over three independent coherent Rician fading channels with equal Rician factor $K=\frac{1}{8}$. Duty factor values are $v=1,0.8,0.5$, and 0.2 .

where, $s^{2}=\sum_{l=1}^{L}\left|d_{l}\right|^{2}$.

When the fading coefficients $\left\{h_{l}\right\}$ are correlated, the average error probability $\bar{P}_{e}$ can be obtained by evaluating the expected value of $P_{e}$ with respect to the joint distribution of $\left(\left|h_{1}\right|, \ldots,\left|h_{L}\right|\right)$, which involves $L$-fold integration. However, if $\left\{\left|h_{l}\right|\right\}$ are Nakagami- $m$ distributed, closed-form expressions for $f_{\chi}(\chi)$ are provided in [19], which lead to a single integration.

Next, we present the simulation results. We define the Rician factor as $K=\frac{|d|^{2}}{\sigma^{2}}$ and correlation coefficient as $\rho=\frac{\operatorname{cov}\left(h_{i}, h_{j}\right)}{\sqrt{\operatorname{var}\left(h_{i}\right) \operatorname{var}\left(h_{j}\right)}}$. Figures 1, 2, and 3 plot the probability of error curves as a function of SNR for 4-OOFSK signaling over Rician fading channels with different number of receiver antennas and different duty factors. Two independent channels are considered in Fig. 1. Note that conventional FSK corre-

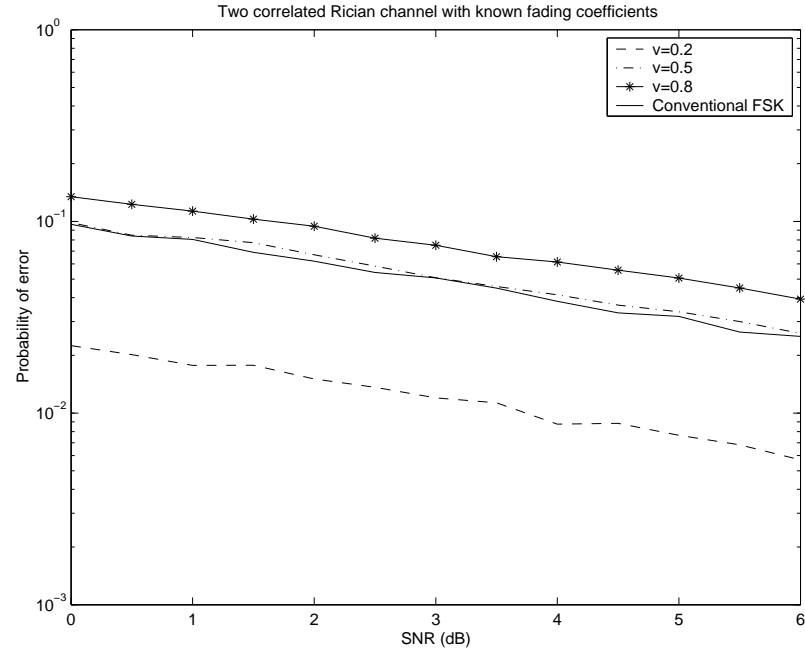

Fig. 3. Error probability vs. SNR for 4-OOFSK signaling over two correlated coherent Rician fading channels with equal Rician factor $K=\frac{1}{8}$ and correlation coefficient $\rho=\frac{1}{4}$. Duty factor values are $v=1,0.8,0.5$, and 0.2 .

sponds to OOFSK with duty factor $v=1$. In Fig. 2, three independent channels are assumed. In both figures, we observe an improvement in the error probability curves if the duty factor $v$ of OOFSK signaling is less than 0.5. When $v=0.2$, we see approximately an order of magnitude improvement in the error performance. This results in substantial energy gains for fixed value of error probability, rendering OOFSK signaling a very energy efficient transmission technique. It can also immediately be noted that due to increased diversity, having more antennas decreases the error rates. In Fig. 3, the case of two correlated channels is investigated. Although the performance is deteriorated due to correlation, OOFSK with sufficiently small duty factor still considerably improves the error performance. It should be noted that having small duty factor means that FSK signals have high peak power but they are transmitted less frequently to satisfy the average power constraint. Consequently, having high peak power signals decrease the error rates.

\section{OOFSK OVER NONCOHERENT FADING ChANNELS}

\section{A. Detection Rule}

In the noncoherent channel case, we assume that the realizations of the fading coefficients $\left\{h_{l}\right\}$ are unknown at both the receiver and transmitter. The receiver is only equipped with the knowledge of the statistics of $\left\{h_{l}\right\}$. We further assume that $\left\{h_{l}\right\}$ are i.i.d. complex Gaussian random variables with $E\left\{h_{l}\right\}=d_{l}$ and $\operatorname{var}\left\{h_{l}\right\}=\sigma^{2}$. Therefore, conditioned on $s_{k}(t)$ being the transmitted signal, $Y_{l, m}$ is a complex Gaussian random variable with

$$
\begin{aligned}
E\left\{Y_{l, m} \mid s_{k}\right\} & =\left\{\begin{array}{ll}
A d_{l} e^{j \theta_{k}} & m=k \\
0 & m \neq k
\end{array},\right. \\
\operatorname{var}\left\{Y_{l, m} \mid s_{k}\right\} & =\left\{\begin{array}{ll}
A^{2} \sigma^{2}+1 & m=k \\
1 & m \neq k
\end{array} .\right.
\end{aligned}
$$


Similarly as in the coherent case, we combine the energies of the $m^{\text {th }}$ frequency components across the antennas, and obtain $R_{m}=\sum_{l=1}^{L} R_{m, l}$. Conditioned on transmitted signal $s_{k}(t)$, $R_{m}$ is a chi-square random variable with the following pdf:

$f_{R_{m} \mid s_{k}}\left(R_{m}\right)= \begin{cases}\frac{1}{\sigma_{y}^{2}}\left(\frac{R_{m}}{\xi}\right)^{\frac{L-1}{2}} e^{-\frac{R_{m}+\xi}{\sigma_{y}^{2}} I_{L-1}\left(\frac{2 \sqrt{R_{m} \xi}}{\sigma_{y}^{2}}\right)} & m=k \\ \frac{R_{m}^{L}-1}{\Gamma(L)} e^{-R_{m}} & m \neq k\end{cases}$

where $\xi=A^{2} \sum_{l=1}^{L}\left|d_{l}\right|^{2}$ and $\sigma_{y}^{2}=A^{2} \sigma^{2}+1$. The vector $\mathbf{R}=\left[R_{1}, \ldots, R_{M}\right]$ has the following conditional joint pdf

$f_{\mathbf{R} \mid s_{k}}(\mathbf{R})=$

$\begin{cases}\frac{1}{\sigma_{y}^{2}}\left(\frac{R_{k}}{\xi}\right)^{\frac{L-1}{2}} e^{-\frac{R_{k}+\xi}{\sigma_{y}^{2}}} I_{L-1}\left(\frac{2 \sqrt{R_{k} \xi}}{\sigma_{y}^{2}}\right) \prod_{n=1}^{M} \quad k \frac{R_{n}^{L-1} e^{-R_{n}}}{\Gamma(L)} & k \neq 0 \\ \frac{1}{[\Gamma(L)]^{M}} \prod_{n=1}^{M} R_{n}^{L-1} e^{-R_{n}} & k=0\end{cases}$

The MAP decision rule that detects $s_{k}$ for $k \neq 0$ is

$$
\left\{\begin{array}{l}
f_{\mathbf{R} \mid s_{k}}>f_{\mathbf{R} \mid s_{m}} \\
f_{\mathbf{R} \mid s_{k}}>\frac{M(1-v)}{v} f_{\mathbf{R} \mid s_{0}}
\end{array} \quad \forall m \neq 0, k\right.
$$

Similarly as in Section $\amalg$, it can be easily shown that

$$
g_{2}\left(R_{k}\right)=R_{k}^{-\frac{L-1}{2}} e^{\frac{R_{k} A^{2} \sigma^{2}}{\sigma_{y}^{2}}} I_{L-1}\left(\frac{2 \sqrt{R_{k} \xi}}{\sigma_{y}^{2}}\right), \quad \xi>0
$$

is a monotonically increasing function. With this observation, the decision rule in (27) simplifies to

$$
\left\{\begin{array}{l}
R_{k}>R_{m} \\
R_{k}>g_{2}^{-1}\left(T_{2}\right)
\end{array} \quad \forall m \neq k\right.
$$

where $T_{2}=\frac{M(1-v) \sigma_{y}^{2} \xi^{\frac{L-1}{2}} e^{\frac{\xi}{\sigma_{y}^{2}}}}{v \Gamma(L)}$. Note that $s_{0}$ is the detected signal if $R_{k}<g_{2}^{-1}\left(T_{2}\right)$ for all $k$.

\section{B. Probability of Error}

We first assume that $s_{1}(t)$ is transmitted. Let $\tau_{2}=g_{2}^{-1}\left(T_{2}\right)$. Then, the probability of correct detection is

$$
P_{c, 1}=P\left(R_{2}>R_{1}, R_{3}>R_{1}, \ldots, R_{M}>R_{1}, R_{1}>\tau_{2} \mid s_{1}\right)
$$

Following an approach similar to that in Section [II], we have

$$
\begin{aligned}
P_{c, 1}= & \sum_{n=0}^{M-1}(-1)^{n}\left(\begin{array}{c}
M-1 \\
n
\end{array}\right) \sum_{i=0}^{n(L-1)} c_{i n} \int_{\tau_{2}}^{\infty} x^{i} e^{-n x} f_{R_{1} \mid s_{1}}(x) d x \\
= & \sum_{n=0}^{M-1}(-1)^{n}\left(\begin{array}{c}
M-1 \\
n
\end{array}\right) \sum_{i=0}^{n(L-1)} c_{i n} \int_{\tau_{2}}^{\infty} x^{i} e^{-n x} \\
& \times \frac{1}{\sigma_{y}^{2}}\left(\frac{x}{\xi}\right)^{\frac{L-1}{2}} e^{-\frac{x+\xi}{\sigma_{y}^{2}}} I_{L-1}\left(\frac{2 \sqrt{x \xi}}{\sigma_{y}^{2}}\right) d x \\
= & \sum_{n=0}^{M-1}(-1)^{n}\left(\begin{array}{c}
M-1 \\
n
\end{array}\right) \sum_{i=0}^{n(L-1)} c_{i n} \frac{\xi^{-\frac{L-1}{2}} e^{-\frac{\xi}{\sigma_{y}^{2}}}}{\sigma_{y}^{2}} \\
& \times\left[\frac{\xi^{\frac{L-1}{2}}(i+L) !}{2\left(1+n \sigma_{y}^{2}\right)^{\frac{i+L}{2}} \sigma_{y}^{L-2-i} L !} F\left(-i, L, \frac{\xi}{\sigma_{y}^{2}\left(1+n \sigma_{y}^{2}\right)}\right)\right. \\
& \times e^{\frac{\xi}{\sigma_{y}^{2}\left(1+n \sigma_{y}^{2}\right)}}-\int_{0}^{\tau_{2}} x^{\frac{2 i+L-1}{2}} e^{-\frac{1+n \sigma_{y}^{2}}{\sigma_{y}^{2}} x} I_{L-1}\left(\frac{2 \sqrt{x \xi}}{\sigma_{y}^{2}}\right) d x
\end{aligned}
$$

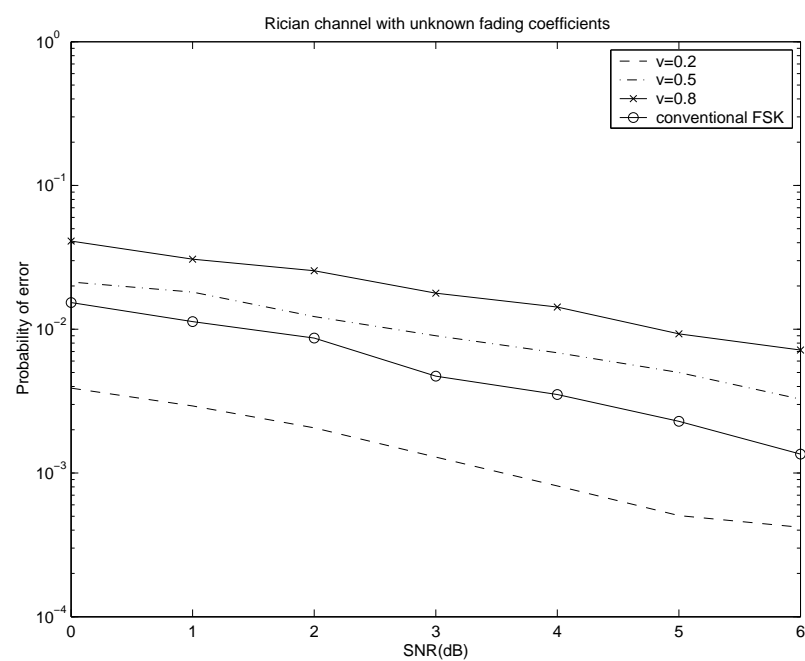

Fig. 4. Error probability vs. SNR for 4-OOFSK signaling over two independent noncoherent Rician fading channels with equal Rician factor $K=\frac{1}{8}$.

If $s_{0}(t)$ is the transmitted signal, the probability of correct detection is

$$
\begin{aligned}
P_{c, 0} & =P\left(R_{1}<\tau_{2}, \ldots, R_{M}<\tau_{2} \mid s_{0}\right) \\
& =\left(1-e^{-\tau} \sum_{l=0}^{L-1} \frac{\tau^{l}}{l !}\right)^{M} .
\end{aligned}
$$

Finally, the average probability of error is

$$
P_{e}=1-\left(v P_{c, 1}+(1-v) P_{c, 0}\right) .
$$

Figures 4, 5, and 6 provide the simulation results of error probability when 4-OOFSK signals are transmitted over noncoherent Rician fading channels. In Figs. 4 and 5, the channels are assumed to be independent. In these figures, it is seen that OOFSK signaling with $v=0.8$ and $v=0.5$ have worse error performance when compared to that of conventional FSK (OOFSK with $v=1$ ). As evidenced in the graph of $v=0.2$, if the duty factor is sufficiently decreased, and hence consequently the peak power is increased, we start seeing improvements. Since fading is not known in the noncoherent case, the advantage of using OOFSK signaling is twofold. Having low duty cycle allows the FSK signals to have high peak power which is especially beneficial when channel characteristics are unknown. In addition, when the zero signal $s_{0}(t)$ is sent, the received signal is composed of additive noise and is free of fading coefficients. Finally, Fig. 6 plots the error probabilities when 4-OOFSK signals are sent over two correlated noncoherent channels. These curves are obtained when the detection rule (29) derived for independent channels are employed at the receiver. We note that the performance degrades due to correlation in noncoherent channels as well. Similar conclusions about OOFSK modulation are drawn.

\section{REFERENCES}

[1] J. R. Pierce, "Ultimate performance of $M$-ary transmissions on fading channels," IEEE Trans. Inform. Theory, vol. IT-12, pp. 2-5, Jan. 1966. 


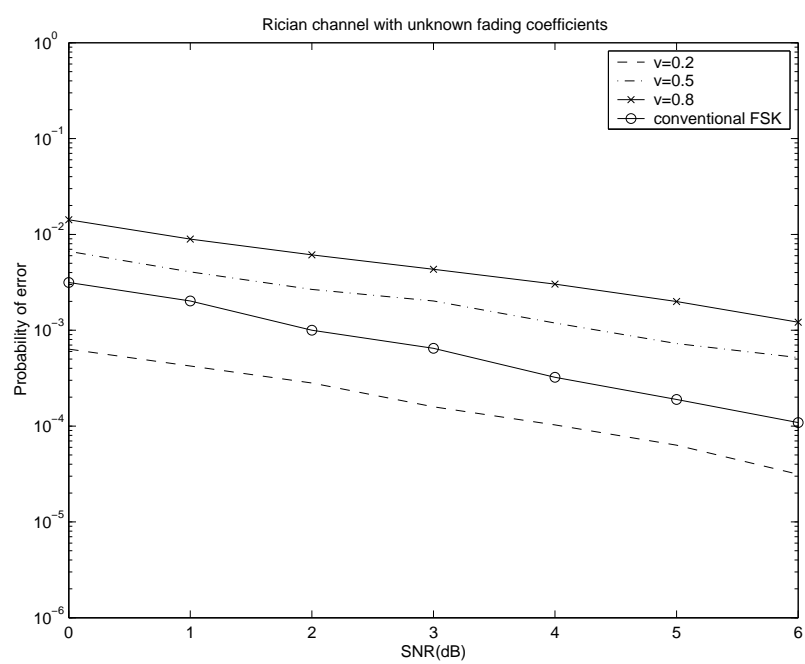

Fig. 5. Error probability vs. SNR for 4-OOFSK signaling over three indepedent noncoherent Rician fading channels with equal Rician factor $K=\frac{1}{8}$.

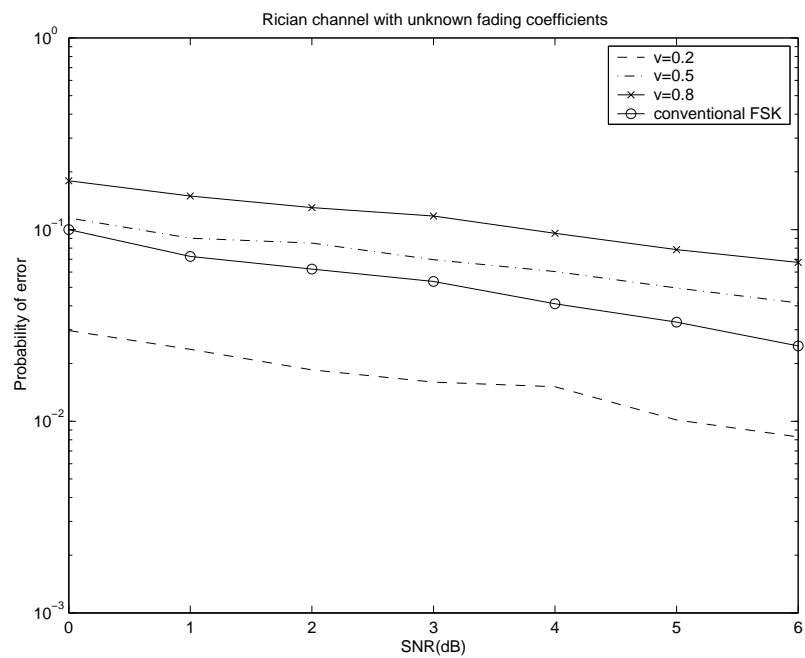

Fig. 6. Error probability vs. SNR for 4-OOFSK signaling over two correlated noncoherent Rician fading channels with equal Rician factor $K=\frac{1}{8}$.

[2] W.C.Lindsey, "Error probabilities for Rician fading multichannel reception of binary and N-ary signals," IEEE Transaction On Information Theory, pp.330-350, October 1964.

[3] J.C.Hancock and W.C.Lindsey, "Optimum performance of self-adaptive communication system operating through a Rayleigh-fading medium," IEEE Transaction On Communication Systems., vol.CS-11, pp.443-454, December 1963

[4] C. W. Helstrom, Statistical Theory of Signal Detection, New York, Pergamon Press, 1968.

[5] S. Verdú, "Spectral efficiency in the wideband regime," IEEE Trans. Inform. Theory, vol. 48, pp. 1319-1343, June 2002

[6] C. Luo and M. Médard, "Frequency-shift keying for ultrawideband Achieving rates of the order of capacity," Proc. 40th Annual Allerton Conference on Communication, Control, and Computing, Monticello, IL, 2002.

[7] D. S. Lun, M. Médard, and I. C. Abou-Faycal, "On the performance of peaky capacity-achieving signaling on multipath fading channels," IEEE Trans. Commun., vol. 52, pp. 931-938, June 2004.

[8] D. S. Lun, M. Médard and I. C.Abou-Faycal, "On performance of peaky capacity-achieving signaling on multipath fading channels," IEEE Transactions On Communications, pp.931-938, vol.52, no.6, Jun 2004.
[9] M. C. Gursoy, H. V. Poor and S. Verdu, "On-Off frequency-shift keying for wideband fading channels," EURASIP Journal on Wireless Communications and Networking, 2006.

[10] Q. Wang and M. C. Gursoy, "Error performance of OOFSK signaling over fading channels," Conference on Information Sciences and Systems(CISS), Princeton University, Princeton, NJ, 2006.

[11] N.L. Johnson and S. Kotz, Continuous univariate distributions, New York, Wiley, 1970.

[12] H. Zhang and T.A. Gulliver, "Error probability for maximum ratio combing multichannel reception for M-ary coherent system over flat Ricean fading channels," IEEE Wireless Communications and Networking Conference, vol. 1, 21-25 Mar 2004, pp.306-310.

[13] D.D.N Bevan, V.T Ermolayev and A.G Flaksman, "Coherent multiple reception of binary modulated signals with dependent Rician fading channel," IEE Proceedings-Communications, Vol.48, Issue 2, April 2001, pp.105 - 111.

[14] V.V. Veeravalli and A. Mantravadi, "Performance analysis for diversity reception of digital signals over correlated fading channels," IEEE Vehicular Technology Conference, vol. 2, 16-20 May 1999, pp.1291 1295.

[15] J. G.Proakis, Digital Communications, New York, McGraw-Hill, 1989.

[16] L. C. Andrews, Special Functions of Mathematics for Engineers, Oxford University Press, 1998.

[17] Y. Chen and N.C.Beaulieu, "SER of selection diversity MFSK with channel estimation errors," IEEE Transactions on Wireless Communications, Vol.5, No.7, July 2006, pp.1920-1928.

[18] M. K.Simon and M.S. Alouini, Digital Communication over Fading Channels, New York, John Wiley \& Sons, 2000.

[19] G. K. Karagiannindis, N. C. Sagias and T.A. Tsiftsis, "Closed-form statistics for the sum of squared Nakagami-m variates and its applications," IEEE Transaction On Communications, vol.54, no.8, pp.13531359, August 2006.

[20] P.Gupta, N,Bansal and R.K. Mallik, "Analysis of minimum selection HS/MRC in Rayleigh channel," IEEE Transaction On Communications, vol.53, no.5, pp.780-784, May 2005. 